\title{
Alignment sensitivity of holographic three-dimensional disks
}

\author{
Hsin-Yu Sidney $\mathrm{Li}^{*}$ and Demetri Psaltis \\ Department of Electrical Engineering, California Institute of Technology, Pasadena, California 91125
}

Received November 3, 1994; revised manuscript received April 10, 1995; accepted April 12, 1995

\begin{abstract}
We describe the rotational alignment sensitivity of three-dimensional holographic disks. It is shown that the reconstructed image always rotates by the angle by which the disk rotates; however, the center and the radius of rotation change as the recording geometry changes. A comparison among image plane, Fourier plane, and Fresnel holograms is given, and an optimum configuration (in terms of alignment sensitivity) in which the radius of rotation is zero is derived. We present experimental results and also discuss how the rotation alignment sensitivity affects the storage density and the readout-recording speed of the three-dimensional disk. A brief summary of other sources of misalignment is given.
\end{abstract}

\section{INTRODUCTION}

The theoretical upper limit on the storage density of volume holograms is $1 / \lambda^{3}$, where $\lambda$ is the operating wavelength of light. This limit is of the order of $10^{12} \mathrm{bits} / \mathrm{cm}^{3}$; however, in practical systems only $10^{9}-10^{10} \mathrm{bits} / \mathrm{cm}^{3}$ is achievable because of the finite numerical aperture of the optical system that transfers the data into the optical system and the dynamic range of the recording medium. For example, typically $10^{3}-10^{4}$ holograms are superimposed at the same location, ${ }^{1,2}$ with each hologram consisting of $10^{3} \times 10^{3}$ pixels, giving a total capacity of $10^{9}-10^{10}$ bits/location. To make them competitive with magnetic and semiconductor memories, which are becoming cheaper and better all the time, it is necessary to further increase the capacity of holographic storage systems. One does this by recording at multiple locations; i.e., by spatial multiplexing. ${ }^{3-7}$ In this paper we address the alignment sensitivity of one of the systems for performing spatial multiplexing, the three-dimensional (3D) holographic disk. . $^{4}$

As in all spatial-multiplexing schemes, the most crucial component of the 3D holographic disk is the scanning mechanism that steers the readout beam to different locations of the disk. In the 3D holographic disk spatial multiplexing is done in a disk configuration, as shown in Fig. 1. The rotation of the disk and the radial motion of the readout head allow us access to any location on the disk surface. Two light beams (a signal and a reference) interfere inside the recording medium to create a grating. Multiple holograms are recorded at the same location by angle multiplexing, ${ }^{8}$ wavelength multiplexing, ${ }^{9,10}$ or phase-code multiplexing. ${ }^{11-13}$ In this paper we concentrate only on angle and wavelength multiplexing. Because of the Bragg-matching requirement of volume holograms, individual holograms can be read out independently by means of a change in direction or the wavelength of the reference beam.

In the holographic data storage system one achieves high readout speed and capacity by reading out entire pages at a time. To convert this information to electronic signals the hologram is imaged onto a de- tector array such as a CCD camera. For the data to be transferred correctly, it is necessary to position the reconstructed holographic image accurately. Inadvertently shifting the reconstructed image by one or even half a pixel (bit) would be disastrous. This is of great concern because the conditions on readout cannot be precisely the same as during recording, and this situation may cause the position of the reconstructed image to change. In addition, because the reference beam is moving relative to the hologram (i.e., the $3 \mathrm{D}$ disk is spinning), this condition will in general also cause the reconstructed image to change over time, causing smearing of the image on readout. It is therefore desirable to reduce the alignment sensitivity for both spatial and angle-wavelength multiplexing.

In this paper we concentrate on the question of rotational alignment sensitivity of 3D holographic disks, which is specific to holographic disk systems. We analyze how rotation affects image reconstruction and discuss how this relates to storage density and recording-readout speed of 3D holographic disks. As is shown below, the reconstructed image always rotates by the same angle at which the disk rotates, with a radius of rotation that depends on various factors. We use this radius (denoted $R_{I}$ ) as a measure of the rotational alignment sensitivity because the larger $R_{I}$ is, the more sensitive the system becomes with respect to rotational errors. A brief discussion of other misalignment errors is given at the end of the paper.

\section{FOURIER-TRANSFORM-TYPE HOLOGRAMS}

The usual configurations for recording holograms can be divided into two categories:

(i) Fourier-transform- (FT-) type holograms: point sources at the input plane [where the spatial light modulator (SLM) is placed] are mapped into plane waves at the recording plane (where the $3 \mathrm{D}$ disk is placed). Such a system is realized by placement of the input plane at the back focal plane of a lens, as shown in Fig. 2. 


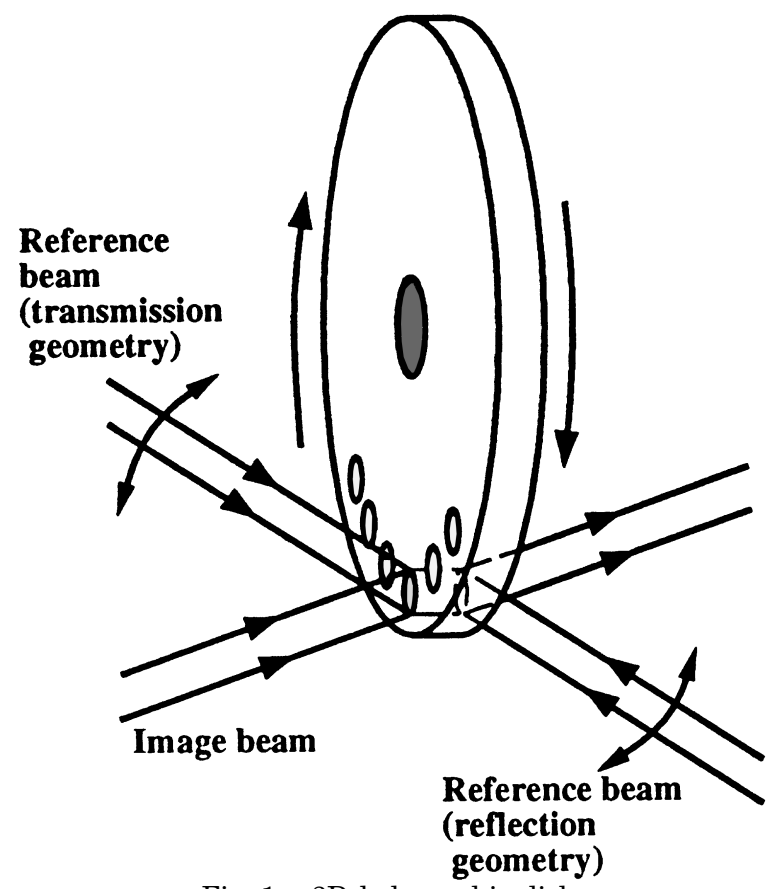

Fig. 1. 3D holographic disk.

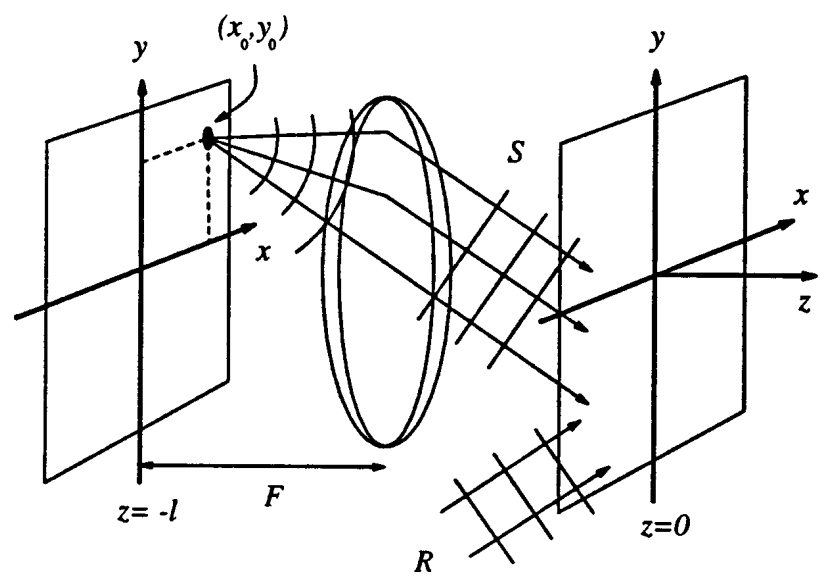

Fig. 2. FT-type holograms have plane-wave impulse responses.

The FT hologram is a special case of this category, with the recording plane being at the FT plane of the lens. In general, the recording plane can be at an arbitrary distance behind the lens. Considered as a linear system, the impulse response is a plane wave.

(ii) Fresnel holograms: point sources at the input plane are mapped into spherical waves at the recording plane, as shown in Fig. 3 below. The impulse response is thus a spherical wave. This category includes imageplane holograms as a special case.

Almost all practical holographic recording systems are equivalent to one of these two cases. The holograms are assumed to be either transmission-type or reflection-type holograms, and the reference beam is assumed to be a plane wave.

In this section we first consider case (i), in which point sources at the input plane give rise to plane waves at the recording plane. We would like to know how the reconstructed image (read out for a hologram) changes as the disk rotates. From this information we can calculate the rotational alignment sensitivity of the 3D disk, which we define as the amount of displacement of the reconstructed image associated with a given amount of hologram or disk rotation.

We approach the problem by considering the situation in which light from a single point source at the input plane interferes with a plane-wave reference beam to record a hologram on the disk. We read out the hologram and see how the reconstructed image of the point source changes as the disk rotates. Assuming that the system is linear (which is true for weak holograms), we can then understand what happens for a general image by superposition. In this section and in Section 3 [in which we analyze case (ii)], we assume that the holograms are essentially planar. In other words, we ignore any Bragg mismatch on hologram readout. Further below we modify the results given in this section and in Section 3 to take into account the volume-holographic nature of the recording medium.

Consider a point source at $\left(x_{0}, y_{0}\right)$ on the input plane. The signal and the reference beams are plane waves of the form (see Fig. 2)

$$
\begin{aligned}
& R=A \exp \left[j k\left(u_{x} x+u_{y} y+u_{z} z\right)\right], \\
& S=B \exp \left[j k\left(v_{x} x+v_{y} y+v_{z} z\right)\right],
\end{aligned}
$$

where $\left(u_{x}, u_{y}, u_{z}\right)$ and $\left(v_{x}, v_{y}, v_{z}\right)$ are the direction cosines of the plane waves. Under the paraxial approximation we have

$$
\begin{aligned}
& v_{x}=x_{0} / F, \\
& v_{y}=y_{0} / F, \\
& v_{z}=\left(1-v_{x}{ }^{2}-v_{y}{ }^{2}\right)^{1 / 2},
\end{aligned}
$$

where $F$ is the focal length of the FT lens. Assuming that the recorded grating strength is proportional to the intensity, the grating recorded at $z=0$ is then proportional to $|R+S|^{2}$ (at $z=0$ ). The part that interests us is the interference term

$$
T=T_{0} \exp \left[j\left(K_{x} x+K_{y} y\right)\right],
$$

where $T_{0}$ is a constant proportional to $A^{*} B$ and where $K_{x}$ and $K_{y}$ are the recorded grating-vector components given by

$$
\begin{aligned}
& K_{x}=k\left(v_{x}-u_{x}\right), \\
& K_{y}=k\left(v_{y}-u_{y}\right) .
\end{aligned}
$$

We now rotate the hologram $T$ around the center of the disk $\left(x_{c}, y_{c}\right)$ and reconstruct the image from the hologram by applying the original reference beam. If $T$ is rotated around $\left(x_{c}, y_{c}\right)$ by $\Delta \phi$, and $\Delta \phi$ is sufficiently small that terms of order $(\Delta \phi)^{2}$ and higher in $\Delta \phi$ can be ignored, then the hologram transmittance $T$ becomes

$$
T^{\prime} \cong T_{0}{ }^{\prime} \exp \left\{j\left[\left(K_{x}-K_{y} \Delta \phi\right) x+\left(K_{y}-K_{x} \Delta \phi\right) y\right]\right\},
$$

where $\left.T_{0}{ }^{\prime}=T_{0} \exp \left[j\left(-K_{x} y_{c}+K_{y} x_{c}\right) \Delta \phi\right)\right]$ is a constant. Illuminating the rotated hologram $T^{\prime}$ with the original reference beam $R$, we find that the reconstructed image is

$$
R T^{\prime} \cong C \exp \left[j k\left(v_{x}{ }^{\prime} x+v_{y}{ }^{\prime} y+v_{z}{ }^{\prime} z\right)\right],
$$

where 


$$
\begin{aligned}
& v_{x}{ }^{\prime}=v_{x}-\left(v_{y}-u_{y}\right) \Delta \phi, \\
& v_{y}{ }^{\prime}=v_{y}+\left(v_{x}-u_{x}\right) \Delta \phi, \\
& v_{z}{ }^{\prime}=\left(1-v_{x}{ }^{2}-v_{y}{ }^{2}\right)^{1 / 2} .
\end{aligned}
$$

Comparing this to the expression for $S$ in Eq. (2) and using Eqs. (3) and (4), we find that the reconstructed image appears to have originated from a point source on the input plane (the back focal plane of the lens in Fig. 2) at

$$
\begin{aligned}
& x_{0}{ }^{\prime}=x_{0}-\left(y_{0}-F u_{y}\right) \Delta \phi, \\
& y_{0}^{\prime}=y_{0}+\left(x_{0}-F u_{x}\right) \Delta \phi .
\end{aligned}
$$

To first order, this result is just the original point source $\left(x_{0}, y_{0}\right)$ rotated by an angle of $\Delta \phi$ about the center at

$$
\begin{aligned}
& x_{c}{ }^{\prime}=F u_{x}, \\
& y_{c}{ }^{\prime}=F u_{y} .
\end{aligned}
$$

Thus, if we record a hologram of an arbitrary image and rotate the hologram by $\Delta \phi$, then by superposition the reconstructed image also rotates by $\Delta \phi$. But instead of rotating around the rotation center of the disk, $\left(x_{c}, y_{c}\right)$, the reconstructed image rotates around the point $\left(x_{c}{ }^{\prime}, y_{c}{ }^{\prime}\right)=$ $\left(F u_{x}, F u_{y}\right)$. Note that $\left(x_{c}{ }^{\prime}, y_{c}{ }^{\prime}\right)$ is independent of the distance $l$ between the disk and the lens (Fig. 2) and depends only on $F$ and on the reference-beam angle. Note also that the apparent direction of motion of the reconstructed image is determined only by the direction of the reference beam relative to the direction of the signal beam and by the disk rotation axis. It is independent of the direction in which the hologram itself is moving.

Because the center of rotation of the reconstructed image may be different from the rotation center of the disk, the radius of rotation of the image (denoted $R_{I}$ ) may also be different from the radius of rotation of the hologram (denoted $R_{H}$ ). For case (i), which we have just discussed, the radius of rotation of the image is

$$
R_{I}=F\left(u_{x}^{2}+u_{y}^{2}\right)^{1 / 2} .
$$

As mentioned in Section 1, we use $R_{I}$ as a measure of the (rotational) alignment sensitivity because, for a small rotation of $\Delta \phi$ in the disk, the amount of displacement in the reconstructed image is $R_{I} \Delta \phi$.

\section{FRESNEL HOLOGRAMS}

We now consider case (ii), in which light from each point source at the input plane is mapped into a spherical wave at the recording plane. Here, as in Section 2, we consider the situation for a single point source at the input plane and see how the reconstructed image changes as the holographic disk rotates. We can then derive the case of an arbitrary image by invoking the superposition principle.

As shown in Fig. 3, the disk is placed at $z=0$ and the input plane is at $z=-l$. Consider the point source at $\left(x_{0}, y_{0},-l\right)$, where $x_{0}$ and $y_{0}$ are much smaller than $l$ (Fig. 3). As before, we ignore the Bragg-matching effect for now. From Fig. 3, the signal and the reference beams are (under paraxial approximations)

$$
\begin{aligned}
R= & A \exp \left[j k\left(u_{x} x+u_{y} y+u_{z} z\right)\right], \\
S= & \frac{B}{z+l} \exp [j k(z+l)] \\
& \times \exp \left\{j \frac{k}{2(z+l)}\left[\left(x-x_{0}\right)^{2}+\left(y-y_{0}\right)^{2}\right\} .\right.
\end{aligned}
$$

We are interested in the interference term of the recorded hologram $|R+S|^{2}($ at $z=0)$ :

$$
T=T_{0}^{\prime \prime} \exp \left\{j \frac{k}{2 l}\left[(x-a)^{2}+(y-b)^{2}\right]\right\}
$$

where $T_{0}$ " is a constant proportional to $\left(A^{*} B / l\right) \exp (j k l)$ $\exp \left\{(k / 2 l)\left[-u_{x} l\left(2 x_{0}+u_{x} l\right)-u_{y} l\left(2 y_{0}+u_{y} l\right)\right]\right\}$ and

$$
\begin{aligned}
& a=x_{0}+u_{x} l, \\
& b=y_{0}+u_{y} l .
\end{aligned}
$$

We now rotate the hologram $T$ around the disk center $\left(x_{c}, y_{c}\right)$ by a small angle $\Delta \phi$ (counterclockwise) and reconstruct the image from $T$ by illuminating the disk with the original reference beam $R$. On rotation the coordinate $(x, y)$ is transformed into $\left(x^{\prime}, y^{\prime}\right)$, where

$$
\begin{aligned}
& x^{\prime}=x-\left(y-y_{c}\right) \Delta \phi, \\
& y^{\prime}=y+\left(x-x_{c}\right) \Delta \phi .
\end{aligned}
$$

Therefore the recorded hologram $T$ becomes

$$
T^{\prime}=T_{0}{ }^{\prime \prime} \exp \left\{j \frac{k}{2 l}\left[\left(x-a^{\prime}\right)^{2}+\left(y-b^{\prime}\right)^{2}\right]\right\}
$$

where $T_{0}{ }^{\prime \prime}$ is the same $T_{0}{ }^{\prime \prime}$ constant as in Eq. (21) and

$$
\begin{aligned}
& a^{\prime}=a-\left(b-y_{c}\right) \Delta \phi, \\
& b^{\prime}=b+\left(a-x_{c}\right) \Delta \phi .
\end{aligned}
$$

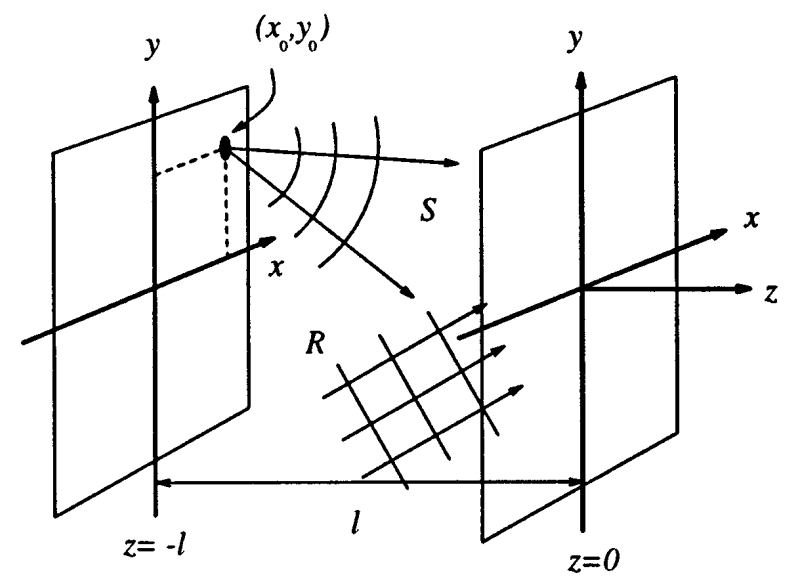

Fig. 3. Fresnel holograms have spherical-wave impulse responses. Image-plane holograms are a special case of Fresnel holograms. 
Illuminating the rotated hologram $T^{\prime}$ [Eq. (26)] with the original reference plane wave $R$, we have, at $z=0$ (immediately after the recording medium),

$$
\begin{aligned}
R T^{\prime}= & A T_{0}{ }^{\prime \prime} \exp \left\{j \frac { k } { 2 l } \left[\left(x-a^{\prime}\right)^{2}+\left(y-b^{\prime}\right)^{2}\right.\right. \\
& \left.\left.+2 l u_{x} x+2 l u_{y} y\right]\right\} \\
= & C \exp \left\{j \frac { k } { 2 l } \left[\left(x-a^{\prime}+l u_{x}\right)^{2}\right.\right. \\
& \left.\left.+\left(y-b^{\prime}+l u_{y}\right)^{2}\right]\right\},
\end{aligned}
$$

where $C=A T_{0}^{\prime \prime} \exp \left\{j(k / 2 l)\left[u_{x} l\left(2 a^{\prime}-u_{x} l\right)+u_{y} l\left(2 b^{\prime}-\right.\right.\right.$ $\left.\left.\left.u_{y} l\right)\right]\right\}$ is a constant. Comparing this result with the expression for the original signal beam $S$ [Eq. (20)], we find that the reconstructed wave $R T^{\prime}$ is a spherical wave that appears to have originated from the point $\left(x_{0}{ }^{\prime}, y_{0}{ }^{\prime},-l\right)$, where

$$
\begin{aligned}
& x_{0}{ }^{\prime}=x_{0}-\left(y_{0}-y_{c}{ }^{\prime}\right) \Delta \phi, \\
& y_{0}{ }^{\prime}=y_{0}+\left(x_{0}-x_{c}{ }^{\prime}\right) \Delta \phi, \\
& x_{c}{ }^{\prime}=x_{c}-u_{x} l, \\
& y_{c}{ }^{\prime}=y_{c}-u_{y} l .
\end{aligned}
$$

Thus, on rotating the disk around its center $\left(x_{c}, y_{c}\right)$ by an angle $\Delta \phi$ (counterclockwise), we find that the reconstructed image of the point source appears to have rotated from $\left(x_{0}, y_{0}\right)$ to $\left(x_{0}^{\prime}, y_{0}^{\prime}\right)$. The apparent center of rotation is therefore $\left(x_{c}{ }^{\prime}, y_{c}{ }^{\prime}\right)$, which is, in general, different from the rotation center of the disk at $\left(x_{c}, y_{c}\right)$. By superposition we conclude that, for any image, when the hologram $T$ rotates by $\Delta \phi$, the reconstructed image will appear to have rotated by the same angle around the center $\left(x_{c}{ }^{\prime}, y_{c}{ }^{\prime}\right)$.

When $l=0$, the hologram is recorded at the image plane. In practice, this is done with an imaging lens (not shown in Fig. 3). In this case $\left(x_{c}{ }^{\prime}, y_{c}{ }^{\prime}\right)$ coincides with $\left(x_{c}, y_{c}\right)$. [Strictly speaking, the paraxial approximation that leads to Eq. (20) does not hold for $l=0$. Nevertheless, the conclusion given above is correct.]

From Eqs. (32) and (33) the radius of rotation of the reconstructed image is

$$
R_{I}=\left[R_{H}^{2}+\left(u_{x}^{2}+u_{y}^{2}\right) l^{2}-2 l\left(x_{c} u_{x}+y_{c} u_{y}\right)\right]^{1 / 2} .
$$

As explained above, we use $R_{I}$ as a measure of the rotational alignment sensitivity because it gives us the amount of displacement in the image per unit angle of rotation of the disk.

In Fig. 4 we show experimental data demonstrating the effect of hologram rotation. Figure 5 shows the configuration used for the experiment. In Fig. 5(a) a point source was recorded as an image-plane hologram on a 5 -mm-thick $\mathrm{LiNbO}_{3}$ disk. Although this is not a planar hologram, for our purpose (assuming small rotation angles) we may treat it as planar. We discuss this subject in more detail in Section 4 and analyze the effects of having a volume hologram instead of a true planar hologram.

We take the origin of the $(x, y)$ coordinate system to be at the center of the hologram recording area on the disk. On rotation of the hologram, the reconstructed image of the point appears to moves horizontally, following the rotation of the hologram along the circumference of the disk [Fig. 4(a)]. We took the images shown in Fig. 4 by making multiple exposures of the reconstructed image of a point source as it moved on rotation of the disk at intervals of $\Delta \phi=0.4^{\circ}$.

In Fig. 5(b) the image of the point was recorded as a FT hologram, where the origin of the $(x, y)$ coordinates is at the center of the hologram recording area on the disk. Figure 4(b) shows the reconstructed images of the point source on rotation of the hologram at $\Delta \phi=$ $0.4^{\circ}$ intervals. Note that the point appears to move vertically even though the hologram itself is essentially moving horizontally. This is because in this case $u_{x} \neq 0$, whereas $u_{y}=0$ (the reference beam is coming in horizontally). From Eqs. (16) and (17) we therefore conclude that the center of rotation of the reconstructed image is on the $x$ axis, away from the origin (the center of the hologram recording region).

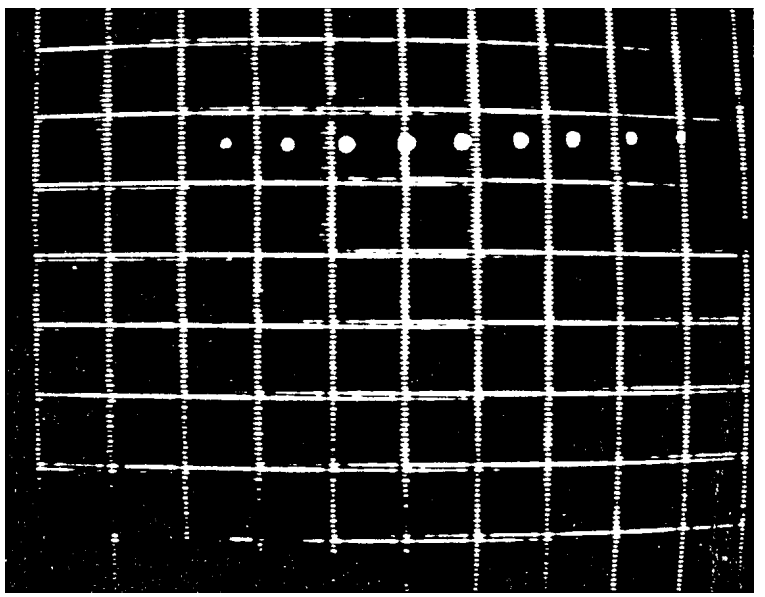

(a)

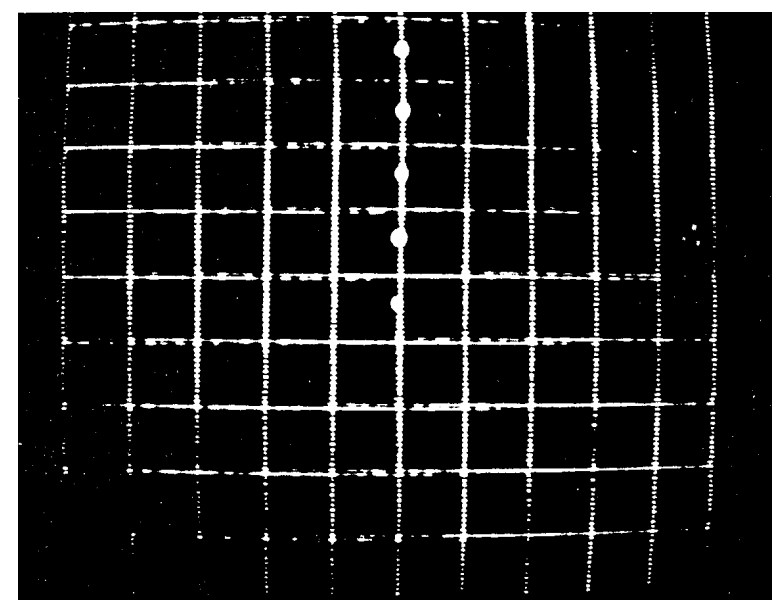

(b)

Fig. 4. Experimental data. Reconstructions of (a) an imageplane hologram by means of the configuration shown in Fig. 5(a) $\left(\Delta \phi=0.4^{\circ}\right)$, (b) a Fourier-plane hologram by means of the configuration shown in Fig. $5(\mathrm{~b})\left(\Delta \phi=0.4^{\circ}\right)$. 


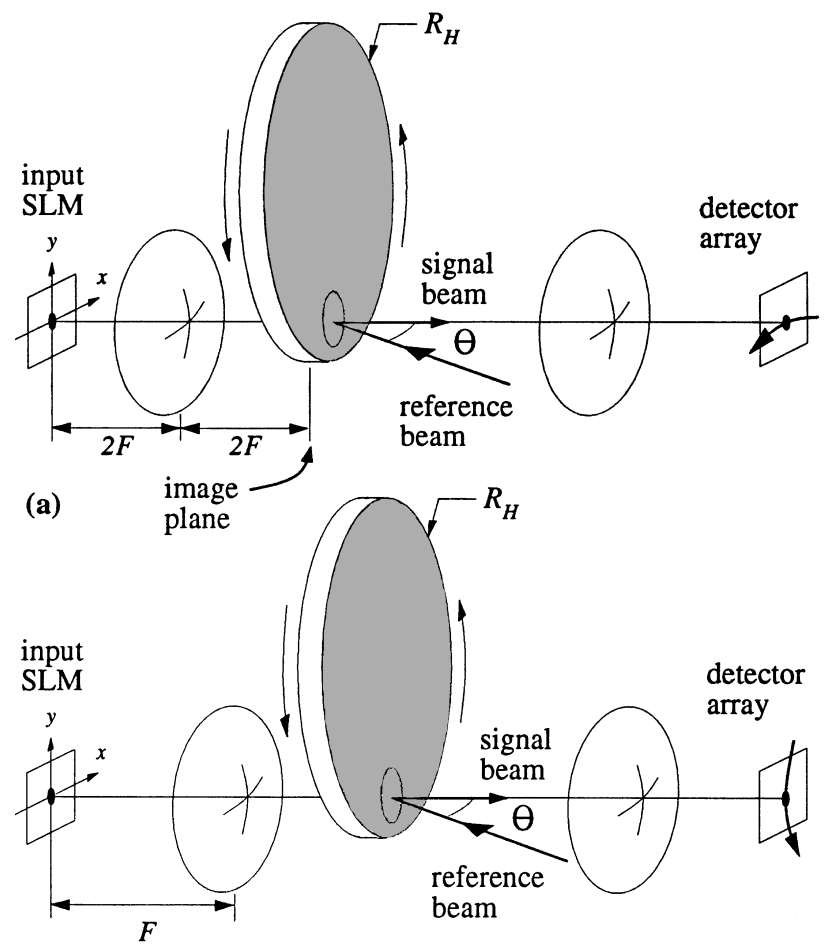

(b)

Fig. 5. Recording geometries for the experimental results shown in Fig. 4: (a) for image-plane holograms $\left[R_{H}=1.7 \mathrm{~cm}\right.$ and $\theta=27^{\circ}$ (outside the crystal)], (b) for Fourier-plane holograms $\left[R_{H}=1.7 \mathrm{~cm}\right.$ and $\theta=27^{\circ}$ (outside the crystal)]. The hologram is $4 \mathrm{~cm}$ before the FT plane.

In general, the direction of motion will be neither horizontal nor vertical but will depend on the distance between the image and the recording media.

\section{EFFECT OF BRAGG MISMATCH ON IMAGE RECONSTRUCTION}

The treatment in Sections 2 and 3 assumes that the holograms are planar, so we may ignore Bragg-matching effects. This is approximately true for sufficiently small rotational angles. For instance, in the experiments described above, disk rotation did not introduce significant Bragg mismatch even though the crystal disk was $5 \mathrm{~mm}$ thick. But when the rotational angle is sufficiently increased we must consider the 3D nature of the hologram. In this section we examine how disk thickness affects the image reconstruction when the holographic disk rotates. We assume that the recorded holograms are weak. This is because when $M$ holograms are superimposed in the same location the diffraction efficiency of each hologram is proportional to $1 / M^{2}{ }^{14}$ In that case we may ignore two-beam coupling effects during recording and secondorder scattering or diffractions during reconstruction.

We start with case (i) (FT-type holograms), in which each point in the input plane corresponds to a plane wave (Fig. 2). We assume that the image is centered on the optical axis. The wave vector of the signal-beam plane wave corresponding to $\left(x_{0}, y_{0}\right)$ at the input plane is [from Eqs. (3) - (5)]

$$
\mathbf{k}_{S} \cong k\left(v_{x}, v_{y}, v_{z}\right)=k\left[\frac{x_{0}}{F}, \frac{y_{0}}{F},\left(1-\frac{x_{0}^{2}+y_{0}^{2}}{F^{2}}\right)^{1 / 2}\right],
$$

where $F$ is the focal length of the FT lens L. The wave vector of the reference-beam plane wave is given by

$$
\mathbf{k}_{R}=k\left(u_{x}, u_{y}, u_{z}\right)=k\left(0,-\sin \theta_{R}, \cos \theta_{R}\right)
$$

where $\theta_{R}$ is the angle between the wave vector and the $z$ axis (so that $u_{x}=0, u_{y}=-\sin \theta_{R}$, and $u_{z}=\cos \theta_{R}$ ). The grating vector is

$$
\begin{aligned}
\mathbf{K}= & \mathbf{k}_{R}-\mathbf{k}_{S}=k\left[-\frac{x_{0}}{F},-\sin \theta_{R}-\frac{y_{0}}{F},\right. \\
& \left.\cos \theta_{R}-\left(1-\frac{x_{0}^{2}+y_{0}^{2}}{F^{2}}\right)^{1 / 2}\right] .
\end{aligned}
$$

When the disk rotates around the $z$ axis by $\Delta \phi, \mathbf{K}$ becomes (to first order in $\Delta \phi$ )

$$
\begin{aligned}
\mathbf{K}^{\prime}= & k\left[-\frac{x_{0}}{F}+\left(\sin \theta_{R}+\frac{y_{0}}{F}\right) \Delta \phi,\right. \\
& -\sin \theta_{R}-\frac{y_{0}}{F}-\frac{x_{0}}{F} \Delta \phi, \\
& \left.\cos \theta_{R}-\left(1-\frac{x_{0}^{2}+y_{0}^{2}}{F^{2}}\right)^{1 / 2}\right] .
\end{aligned}
$$

When the hologram is illuminated with the original reference beam a plane wave is reconstructed. To determine the direction and the amplitude of this diffracted plane wave, we first form the wave vector

$$
\begin{gathered}
\mathbf{k}_{S}^{\prime}=\mathbf{k}_{R}-\mathbf{K}^{\prime}=k\left[\frac{x_{0}}{F}-\left(\sin \theta_{R}+\frac{y_{0}}{F}\right) \Delta \phi,\right. \\
\left.\frac{y_{0}}{F}+\frac{x_{0}}{F} \Delta \phi,\left(1-\frac{x_{0}^{2}+y_{0}^{2}}{F^{2}}\right)^{1 / 2}\right] .
\end{gathered}
$$

The magnitude of $\mathbf{k}_{S}$ ' is, in general, not equal to $k=$ $2 \pi n / \lambda$ (where $n$ is the index of refraction of the recording medium). What happens is that the wave vector of the actual diffracted plane wave has the same $x-y$ components as $\mathbf{k}_{S}{ }^{\prime}$ but a different $z$ component, so that its length is equal to $k$ (the normal to the surface of the recording medium is along the $z$ direction). The difference between the $z$ components of $\mathbf{k}_{S}{ }^{\prime}$ and the wave vector of the actual diffracted plane wave is the Bragg mismatch (Fig. 6). ${ }^{15,16}$ The intensity of the diffracted plane wave depends on the amount of Bragg mismatch and on the thickness of the recording medium. For planar holograms the thickness is negligible (by definition), and there is negligible change in the intensity as a result of Bragg mismatch. This is exactly what was done in Sections 2 and 3, where we considered just the $x-y$ components. Thus, for weak holograms, the intensity of the diffracted plane waves depends on the amount of Bragg mismatch of the diffracted wave vector, but the direction is not affected and is the same as if the recording medium were planar. To correct for the 3D nature of the holograms in Sections 2 and 3, we therefore need only 


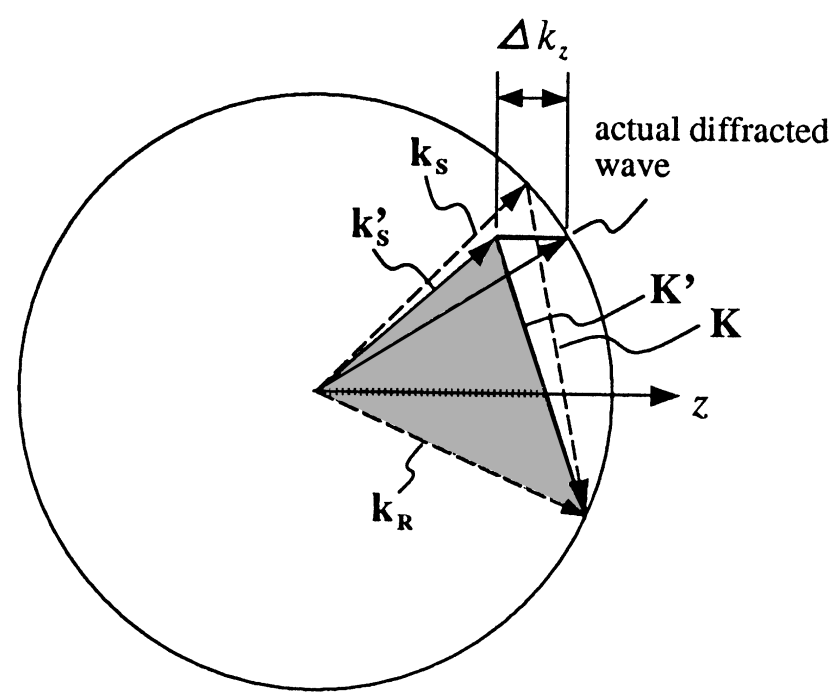

Fig. 6. Bragg-matching representation in $k$ space.

consider the modification in amplitude of the diffractedplane-wave components from the hologram. For case (i), the plane-wave components correspond to point sources at the input plane. The modification in the reconstructed image intensity is therefore a pointwise effect.

From the geometry of Fig. 6, to first order in $\Delta \phi$, the Bragg mismatch along the $z$ direction is

$$
\Delta k_{z} \cong \frac{k x_{0}}{F} \sin \theta_{R} \Delta \phi,
$$

and the amplitude of the readout plane wave is proportional to ${ }^{15,16}$

$$
\operatorname{sinc}\left(\frac{L}{2 \pi} \Delta k_{z}\right)=\operatorname{sinc}\left(\frac{k L x_{0}}{2 \pi F} \sin \theta_{R} \Delta \phi\right),
$$

where $L$ is the thickness of the recording material and $\operatorname{sinc}(x)$ is defined here as $\sin \pi x / \pi x$.

When $\Delta \phi=0$, the value of the sinc function is equal to 1 , and the entire image is reconstructed uniformly as expected. As $\Delta \phi$ increases, the width of the sinc function (as a function of $x_{0}$ ) becomes narrower, and the points farther from the center (larger $x_{0}$ ) show a greater decrease in intensity because of the sinc function. For the center of the image, $x_{0}$ is zero (note that this is not the center of the original image), and therefore the value of the sinc function is 1 for any rotation $\Delta \phi$. Note that, because the argument of the sinc function depends only on $x_{0}$, but not on $y_{0}$, the intensities along strips parallel to the $y$ axis are the same.

For case (ii) (Fresnel holograms), the situation is slightly more complicated. Recall that for Fresnel holograms the plane-wave components at the recording plane correspond to the FT components of the image, whereas for FT-type holograms the plane-wave components at the recording plane correspond to points of the image. Thus we can still use the results derived above, but instead of considering point sources at the input plane we now consider the plane-wave components that make up the image. To use these results, we note that for the Fresnel hologram a plane-wave component corresponding to spatial frequency $w_{x}$ (Fig. 3 ) has the same wave vector as does a plane-wave originating from a point source at position $x=\lambda F w_{x}$ in the FT-type hologram (Fig. 2). We therefore substitute $\lambda F w_{x}$ for the variable $x_{0}$ in Eq. (41). The sinc factor now becomes

$$
\operatorname{sinc}\left(L w_{x} \sin \theta_{R} \Delta \phi\right)
$$

In this case the result of Bragg mismatch is not a pointwise effect but a modification in the spatial frequencies. For small $\Delta \phi$ the sinc factor cuts off the higher spatial frequencies. However, as the rotation angle $\Delta \phi$ becomes larger, the FT of the reconstructed image starts to shift in a manner identical to that by which the reconstructed image shifts in the case of FT-type holograms. At the same time, the width of the sinc function (as a function of $w_{x}$ ) decreases. When $\Delta \phi$ is large enough, the central lobe of the sinc function becomes very narrow, whereas the FT of the reconstructed image shifts because of disk rotation. This effect is analogous to the image shift in the FT-type holograms discussed above. As a result, we obtain a reconstruction whose spatial spectrum has no dc component. In effect, the reconstructed image will appear to be edge enhanced. From expression (42) the width of the central lobe of the sinc function is

$$
\Delta x_{0}=\frac{2 \lambda F}{L \sin \theta_{R} \Delta \phi},
$$

whereas the shift of the spectrum is

$$
\Delta x_{w}=F \sin \theta_{R} \Delta \phi \text {. }
$$

Therefore we expect the edge-enhancement effect to become observable when $\Delta x_{w}=\Delta x_{0} / 2$, or when

$$
\Delta \phi=\sqrt{\frac{\lambda}{L}} \frac{1}{\sin \theta_{R}} .
$$

Note that this scales as the square root of $\lambda / L$, whereas the usual angle selectivity of volume holograms scales linearly with $\lambda / L$. (Note that, in the formula above, both $\theta_{R}$ and $\lambda$ are the values measured inside the holographic medium). For example, for $\lambda=488 \mathrm{~nm}$ (in vacuum), $L=5 \mathrm{~nm}, n=2.2$ (refractive index for lithium niobate), and $\theta_{R}=30^{\circ}$ (outside the recording medium), the wavelength inside the crystal is $\lambda=221.8 \mathrm{~nm}$, and the angle inside the crystal is $\theta_{R}=13^{\circ}$. This result gives us a $\Delta \phi$ value of roughly $1.5^{\circ}$, which is approximately what we measured in the experiment discussed in Fig. 7(a), shown and described below.

For Fresnel holograms, besides the Bragg-mismatching effect there is also an effect that comes from the fact that the radius of rotation $R_{I}$ [Eq. (34)] depends on $l$ (the distance from the point source to the recording plane). Because this parameter changes throughout the thickness of the recording material for volume holograms, $R_{I}$ is not a constant. The effect is that (even if there were no Bragg-matching problems) the reconstructed image starts to blur out as the rotational angle becomes larger. However, if $l$ is much larger than the thickness of the hologram, then the Bragg-mismatching effect dominates. [Note that this not a problem for FT-type holograms because $R_{I}$ in Eq. (18) does not depend on the distance between the recording plane and the FT lens.] 


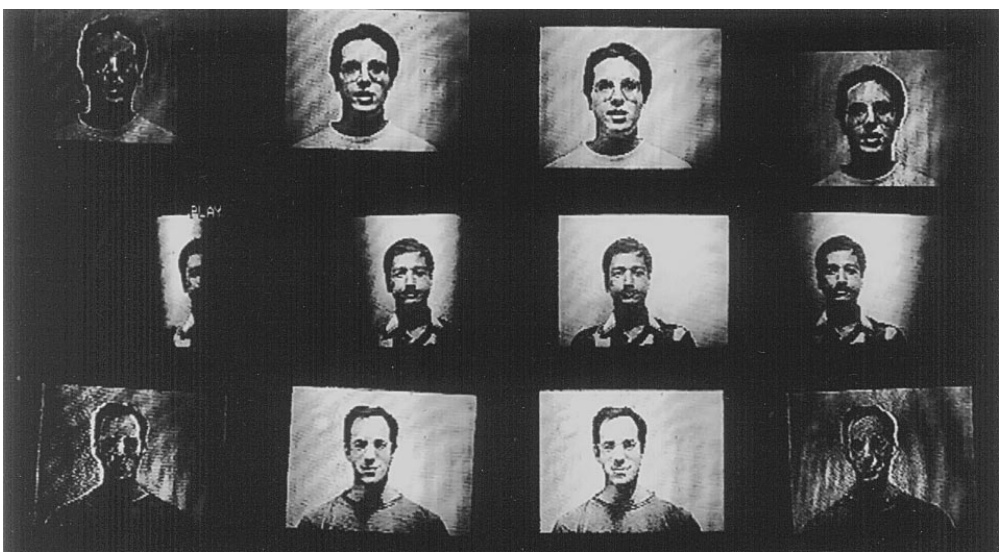

Fig. 7. Reconstructed images from rotated holograms: top row, rotation of an image-plane hologram; middle row, rotation of a Fourier-plane hologram; bottom row, rotation of a hologram recorded in the optimum configuration for minimizing alignment sensitivity.
The predictions of the effects of Bragg selectivity on image reconstruction are demonstrated by the experimental results shown in Fig. 7 . We show the effect of hologram rotation on the reconstruction of recorded images stored as (top row) image-plane holograms, (middle row) Fourier-plane holograms, and (bottom row) optimumconfiguration holograms, which is a special case of the Fresnel hologram (we further discuss this subject in Section 5). The experimental setup is the same as that used previously: we use the setup shown in Fig. 5(a) for the image-plane hologram and the setup shown in Fig. 5(b) for the FT hologram. The setup for the optimum configuration is similar to that shown in Fig. 5(a), except the distance between the recording plane and the imaging lens is changed. This result is explained in more detail in Section 5. In the experiment the CCD camera and the input SLM were both rotated $90^{\circ}$, so that the horizontal direction in the picture is actually vertical in the setup.

In all cases the reconstructed image rotates as the holographic disk rotates. The results are similar to the experimental results shown in Fig. 4: for image-plane holograms the reconstructed image moves in the same direction as the disk, whereas for Fourier-plane holograms the direction is perpendicular to the direction of motion of the disk. The effects of Bragg mismatch are apparent in Fig. 7. For Fourier-plane holograms, as the disk rotates, the visible part of the image becomes narrower and is centered around the head of the person. Eventually, of course, the hologram rotates outside the region of the reference-beam illumination, and the image disappears. For image-plane holograms and optimum-configuration holograms (both special cases of Fresnel holograms), the reconstructed image starts to show edge-enhancement effects as the rotation angle becomes larger, as predicted.

\section{OPTIMUM CONFIGURATION}

In either case-FT-type or Fresnel holograms-the reconstructed image will appear to rotate around some center by the same angle that the disk rotates. As shown in Section 4, Bragg selectivity will affect image reconstruction, but the amount of rotation is still correctly described by the planar hologram analysis. As shown by the experimental results given in Fig. 7 , there is a considerable amount of image motion before the image disappears because of Bragg mismatch. For the purpose of data readout, we would like to minimize the amount of this displacement to avoid smearing the detected image while maximizing the allowable detector integration time.

Because the reconstructed image always rotates by the same amount as the disk, the best that can be done to minimize displacement is to have the center of rotation at the center of the reconstructed image itself. From Eqs. (32) and (33) we can do this by setting

$$
\begin{aligned}
& x_{c}=u_{x} l, \\
& y_{c}=u_{y} l .
\end{aligned}
$$

It is assumed here that the center of the image is at $x=0$, $y=0$ (if this is not true, then the conditions given above must be rewritten in the shifted coordinate system, but the idea is the same). For example, in Fig. 8, $y_{c}=0$ and $u_{y}=0$. Given $x_{c}$ and $u_{x}$, the distance $l$ that gives the optimum configuration can be determined by Eq. (46). In general, given $x_{c}$ and $y_{c}$, we select a convenient distance $l$, and these values determine the optimum referencebeam direction $u_{x}$ and $u_{y}$. In this optimum configuration (which was mentioned in Section 4 in connection with the bottom row of Fig. 7), the pixels at the edge of the image will move the most, and for the worst case the radius of rotation is $r$, where $r$ is the distance from the center of the image to the outermost pixel. In this case the alignment sensitivity is $R_{I}=r$. This result can be much less than the actual radius of rotation of the hologram, $R_{H}$.

Of course, once $l$ (the distance between the image plane and the disk) and $\left(x_{c}, y_{c}\right)$ (the disk center) are fixed, there is only one reference-beam angle that will give the optimum configuration. For angle multiplexing in which the

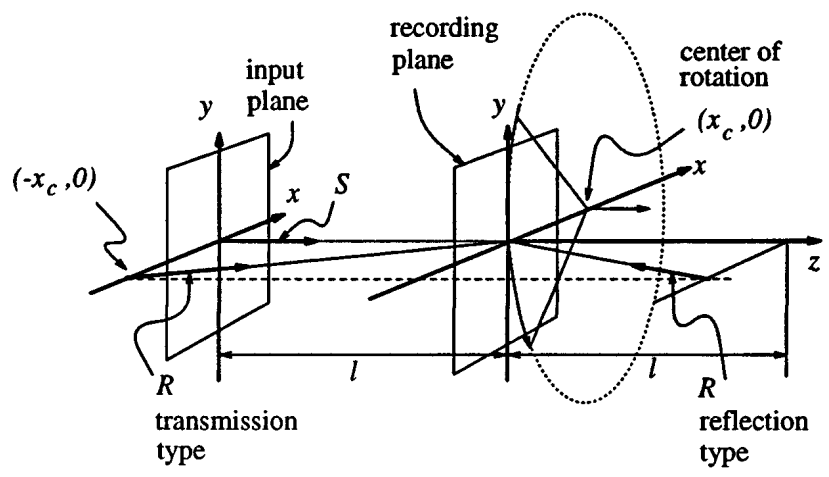

Fig. 8. Optimum recording configuration system with minimum rotational alignment sensitivity. 
reference-beam angle changes, we would set the center of the reference-beam angle swing to be at the optimum angle. For wavelength multiplexing, the angle of the reference beam need not be changed.

The bottom row of Fig. 7 shows the effect of hologram rotation on the reconstruction of recorded images stored as an optimum-configuration hologram. Note that for the optimum configuration, although the image still rotates and displays the edge-enhancement effect, it rotates around the center of the image instead of moving out of the field of view.

\section{STORAGE DENSITY AND ALIGNMENT SENSITIVITY}

Having derived the 3D disk's alignment sensitivity $\left(R_{I}\right)$ resulting from rotational misalignment, we would like to compare the alignment sensitivity of the optimum configuration with image-plane holograms and Fourier-plane holograms, which are the most commonly used. First, however, we must consider under what conditions this comparison is to be conducted. Because we are interested in using 3D holographic disks for data storage, we choose to compare the three systems under the same maximum storage density. ${ }^{7,15}$

In Sections 2-5 we showed that there is an optimum configuration for minimum rotational alignment sensitivity. This result would seem to be incompatible with the condition for maximizing the storage capacity of a 3D disk, which requires that the disk be at the image plane or the Fourier plane. ${ }^{7}$ In fact, however, it is possible to satisfy the conditions for both minimum rotational alignment sensitivity and maximum storage density simultaneously. In this section we describe how this can be done.

The configuration shown in Fig. 9 was proposed by VanderLugt for recording planar holograms. ${ }^{17}$ The SLM with transmittance $f(x, y)$ is placed at $\mathrm{P} 1$, immediately after condenser lens L1. When illuminated with a plane wave, condenser lens L1 provides a converging spherical wave that illuminates the SLM and produces the FT of $f(x, y)$ (with an additional quadratic phase) at the back focal plane (P2). The FT of $f(x, y)$ can then be recorded as a hologram with a reference plane wave. A second lens, L2, placed immediately after the hologram at P2 is used to form the (inverted) image of $f(x, y)$ at image plane P3. The distances between the lenses are determined by the familiar imaging condition

$$
\frac{1}{F_{1}}+\frac{1}{l_{2}}=\frac{1}{F_{2}}
$$

where $F_{1}$ is the focal length of condenser lens L1 and $F_{2}$ is the focal length of lens L2. In VanderLugt's paper ${ }^{17}$ the holograms were assumed to be planar. The system is of course also usable for volume holograms.

For the system we have just described (which we refer to as the VanderLugt configuration), point sources on the SLM at P1 correspond to spherical waves. Therefore it is possible to design the system according to the specifications of Section 5 to set the center of rotation of the hologram at the center of the reconstructed image by setting $F_{1}=l$. At the same time, we show below that it is also possible to achieve maximum storage density with this system.

First, consider the spatial extent of the hologram in the VanderLugt system. Let $u_{\max }$ be the highest spatial frequency of $f(x, y)$. Then the (spatial) extent of the FT at P2 (within paraxial approximations) is $a=2 u_{\max } \lambda F_{1}$. Because all the information of the original image $f(x, y)$ is contained within this region, this is the minimum aperture required for recording the hologram (and for using lens L2). If the pixel spacing of the SLM is $\delta$, and $N_{p}$ is the number of pixels along one dimension, then $u_{\max }=1 / \delta$, and we can write $a$ as

$$
a=\frac{2 \lambda F_{1}}{\delta}=2 \lambda N_{p}\left(\frac{F_{1}}{N_{p} \delta}\right)=\lambda N_{p}(F / \#),
$$

where $F$ /\# is the $f$-number of the lens, assuming that the aperture of L1 is the same as the size of SLM (which is $\left.N_{p} \delta\right)$. Thus, if the ratio $(F / \#)=F_{1} / N_{p} \delta$ is fixed, $a$ is a constant even when the image size $N_{p} \delta$ changes (assuming that $F_{1}$ changes accordingly).

Let us compare the VanderLugt configuration with the conventional 4-F FT system (Fig. 10), which we used previously to achieve maximum storage density. ${ }^{7}$ If the focal length of FT lens $\mathrm{L}^{\prime}$ is also $F_{1}$, then the spatial extent of the FT is also $a=2 \lambda N_{p}\left(F_{1} / N_{p} \delta\right)$. Therefore, no matter what spatial extent of the hologram is required for achieving maximum storage density in the $4-F$ system, the same recording area can be obtained equally well with the VanderLugt system

The VanderLugt configuration, however, has some additional advantages over the conventional $4-F$ system. First, the lens aperture of $\mathrm{L}^{\prime}$ must be larger than $N_{p} \delta$ (the aperture of L1) to allow all the spatial frequencies from $f(x, y)$ to pass through. In addition, the aberration corrections for $\mathrm{L}^{\prime}$ are considerably more complicated

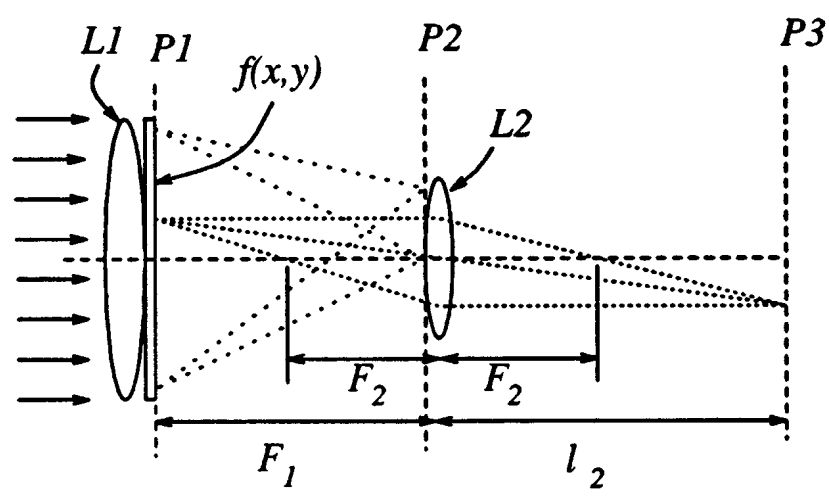

Fig. 9. VanderLugt imaging system.

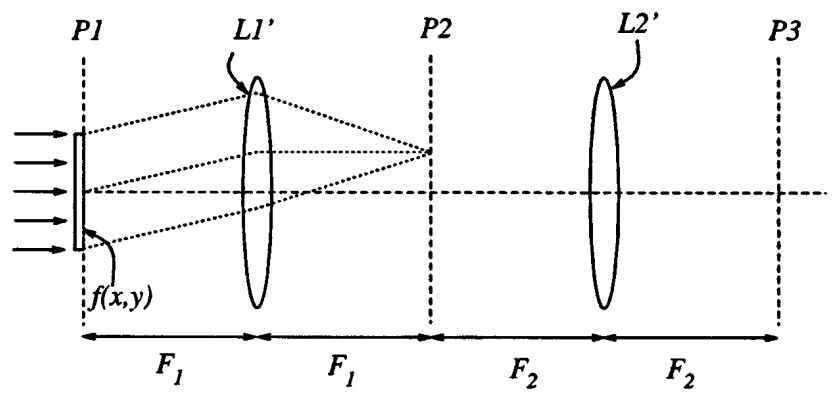

Fig. 10. Conventional 4- $F$ imaging system. 
than for condenser lens L1, which need only produce a good converging spherical wave (a practical implementation would probably use an aspherical L1 lens). Therefore the requirements on $\mathrm{L} 1$ are easier than for $\mathrm{L} 1^{\prime}$. The requirements for $\mathrm{L} 2^{\prime}$ are similar to those for $\mathrm{L} 1^{\prime}$ and are more stringent than for L2 or L1.

\section{ALIGNMENT-LIMITED MAXIMUM READOUT TIME}

Given the rotational speed of the disk, we calculate in this section how long the reconstructed image stays within half a pixel. This is the largest allowable detector integration time that we may use to read out the hologram if the disk is continuously spinning. This time will depend on the way the holograms are stored (e.g., as FT holograms or image-plane holograms).

First we consider image-plane holograms. Let $\delta$ be the pixel spacing at the image plane inside the recording medium, and let $R$ be the radius of rotation, i.e., the distance from the center of the hologram to the disk rotation axis. If the disk rotates by a small angle $\Delta \phi$, then the image will rotate by the same angle and will move a distance of $R \Delta \phi$. For this value to be less than half the pixel spacing, we require that

$$
\Delta x=R \Delta \phi \leq(1 / 2) \delta .
$$

If $T$ is the period of rotation of the disk, then the time in which the reconstructed image remains within half a pixel spacing is

$$
\tau=\frac{T}{2 \pi} \Delta \phi \leq \frac{T}{2 \pi} \frac{\delta}{2 R}=\frac{T}{2 \pi N_{p}}\left(\frac{N_{p} \delta}{2 R}\right) .
$$

Note that $\tau$ is independent of image magnification (the actual size of the image at the readout plane is, in general, different from the image size inside the recording medium). The value of the pixel spacing $\delta$ is chosen for achievement of maximum storage density, as derived in Ref. 7.

Next we consider the optimum configuration. Because the reconstructed image rotates around the center of the image at the same rate as the disk, the center pixels of the image move very little, and the pixels that are displaced the most are the outermost pixels. Because the image size is $\left(N_{p} \delta\right) \times\left(N_{p} \delta\right)$, the most that the pixels will move when the disk rotates by $\Delta \phi$ is $(1 / 2) N_{p} \delta \Delta \phi$. For this value to be less than $\delta / 2$, we have

$$
\Delta \phi \leq \frac{1}{N_{p}},
$$

and the maximum amount of time in which the hologram can be read out is

$$
\tau=\frac{T}{2 \pi N_{p}}
$$

It is interesting that this result is independent of pixel spacing $\delta$ and also applies to the VanderLugt recording configuration.

For Fourier-plane holograms, we assume normal incidence for the signal beam. Let $\theta$ be the angle between the reference beam and the normal to the hologram surface. When the hologram rotates by $\Delta \phi$, the grating also rotates by the same amount. In this case the reconstructed wave vector tilts by an angle of $\sin \theta \Delta \phi$, and the reconstructed image of the pixel will therefore appear to move by

$$
\Delta y=F \Delta \phi=F \sin \theta \Delta \phi
$$

where $F$ is the focal length of the FT lens. It is easy to verify that this result is consistent with the result given by Eq. (18). In contrast, the spatial extent of the FT hologram is $2 \lambda F / \delta^{\prime}$, where $\delta^{\prime}$ is the pixel size of the image. For optimum storage density the spatial extent of the FT should be the same as $N_{p} \delta$, where $\delta$ is the optimum pixel size for obtaining maximum storage density in the case of image-plane holograms. Thus

$$
N_{p} \delta=2 \lambda F / \delta^{\prime}
$$

The optimum pixel size for FT holograms $\delta^{\prime}$ is therefore related to the optimum pixel size of image-plane holograms $\delta$ by the equation

$$
\delta^{\prime}=\frac{2 \lambda F}{N_{p} \delta}
$$

For $\Delta y$ to be less than half of $\delta^{\prime}$, we require that

$$
\Delta \phi \leq \frac{\lambda}{N_{p} \delta \sin \theta}
$$

The maximum readout time is therefore

$$
\tau=\frac{T}{2 \pi N_{p}}\left(\frac{\lambda}{\delta \sin \theta}\right)
$$

which is independent of the focal length $F$.

Note that, although the displacement for the reconstructed image for the image-plane hologram, $\Delta x$ [inequality (50)], and that for the FT hologram, $\Delta y$ [Eq. (56)], are approximately the same, the pixel sizes with which they are compared (i.e., $\delta$ and $\delta^{\prime}$ ) are very different. For FT holograms to have the same storage density as image-plane holograms, the spatial extent of the FT hologram must be small. This requirement implies that the pixel size of the image is large. Thus, although $\Delta x$ and $\Delta y$ may be approximately the same, the pixel size of the image-plane hologram $\delta$ is, in general, much smaller than $\delta^{\prime}$.

\section{NOISE-LIMITED MINIMUM READOUT TIME}

The next question that we address is whether the maximum available detector integration time that is due to rotation is sufficient for actual holographic readout by detectors. In this section we compare it with the estimated noise-limited minimum readout time.

By noise-limited minimum readout time we mean the time that it takes for a detector array to accumulate enough photons from the light diffracted by the hologram for the detector signal to rise significantly above the noise 
level. A typical commercially available CCD camera has a noise-equivalent exposure of approximately $45 \mathrm{pJ} / \mathrm{cm}^{2}$. For an operating wavelength of $\lambda=500 \mathrm{~nm}$, we have approximately $4 \times 10^{-19} \mathrm{~J} /$ photon. Thus each pixel requires roughly 26 photons for generating a signal equivalent to detector noise. Let $M$ be the number of photons required for detection; $\eta$, the diffraction efficiency; $N_{p}{ }^{2}$, the number of pixels; and $P_{\text {inc }}$, the reference-beam power. If the time it takes to accumulate $M$ photons/pixel is $\tau$, then

$$
\frac{\eta P_{\text {inc }}}{N_{p}{ }^{2}} \tau=\frac{M h c}{\lambda},
$$

where $h$ is Planck's constant and $c$ is the speed of light. This equation gives us

$$
\tau=\frac{M h c N_{p}^{2}}{\eta P_{\text {inc }} \lambda},
$$

and the maximum data transfer rate is

$$
\frac{N_{p}^{2}}{\tau}=\frac{\eta P_{\text {inc }} \lambda}{M h c}
$$

As an example, let the following parameters apply:

Number of pixels per page: $N_{p}=500 \times 500$,

Readout reference-beam power: $P_{\text {inc }}=200 \mathrm{~mW}$, Minimum number of photons required: $M=500$, Diffraction efficiency: $\eta=10^{-5}$,

Wavelength: $\lambda=500 \mathrm{~nm}$.

In this case we have a transfer rate of $10 \mathrm{Gbits} / \mathrm{s}$ and a detector integration time (i.e., readout time) of $\tau=$ $25 \mu \mathrm{s}$. The integration time must be less than the time in which the reconstructed image moves by half a pixel as a result of disk rotation, as described in Section 7 . This requirement puts a limit on how fast we may spin the disk. Let the following parameters also apply:

Optimum pixel size: It can be shown ${ }^{7,15}$ that, for an $f / 3$ lens, $\delta \approx 3 \mu \mathrm{m}$. This value is for the image-plane hologram; the corresponding optimum pixel size for the Fourier-plane hologram is $\delta^{\prime}=33 \mu \mathrm{m}$, assuming that $F=10 \mathrm{~cm}$.

Radius of rotation: $R=50 \mathrm{~mm}$.

Reference-beam angle: $\theta=15^{\circ}$ (inside the holographic recording material).

We calculate the maximum allowable disk rotation speed to be

Image plane: $11 \mathrm{rpm}(T=5 \mathrm{~s})$, Fourier plane: $490 \mathrm{rpm}(T=120 \mathrm{~ms})$, Optimum configuration: $763 \mathrm{rpm}(T=79 \mathrm{~ms})$.

The maximum allowable rotation speeds for Fourier-plane holograms and the optimum-configuration holograms are approximately the same, whereas for image-plane holograms the integration time is much lower.

The data transfer rate depends on how fast we can read out the holograms, whereas random access time (which is proportional to the period of disk rotation, $T$ ) depends on how fast we spin the disk. It is interesting that (assuming that all other factors remain the same), as we decrease the SLM pixel array size, $N_{p}$, the minimum required detector integration time decreases, but the data transfer rate does not change. At the same time, because the integration time has decreased, the maximum allowable disk speed goes up, which means that we may spin the disk at a high rate and lower the random access time. In contrast, as $N_{p}$ goes down, so does the storage density. ${ }^{7}$ Thus there is a trade-off between storage density and random access time.

\section{DISCUSSION AND CONCLUSIONS}

In discussing the rotational alignment sensitivity, we have thus far assumed that the reference beam is a plane wave. One might ask whether using some other waveform as the reference beam would make any difference in terms of rotational alignment sensitivity.

The next simplest possible wave form is the spherical wave. It turns out, however, that, when spherical-wave reference beams are used, the reconstructed image from the hologram still rotates by the same amount of angle as the hologram itself. ${ }^{16}$ What changes is the center of rotation relative to the reconstructed image and the radius of rotation. The analysis (assuming paraxial approximations) is similar to the two cases (viz., FT-type holograms and Fresnel holograms) discussed above and will not be repeated here.

In applying the results derived above, we should take into account refraction at the surface of the recording media. In particular, we should first change angles from outside to inside the recording medium. Roughly speaking, when seen from inside the recording medium, distances from the object to the surface of the recording medium will appear to be $n$ times the actual distance (under paraxial approximations), where $n$ is the refractive index of the recording medium. Thus the focal length $F$ of the lens should be replaced by $n F$, and so on.

In this paper we have concentrated on rotation alignment sensitivity, which is specific to the $3 \mathrm{D}$ disk. In general, for any holographic memory system that uses spatial and angle-wavelength multiplexing, the types of misalignment may be summarized in five categories:

(i) Error in reference-beam angle,

(ii) Error in reference-beam wavelength,

(iii) Hologram translation,

(iv) Hologram tilt,

(v) Hologram rotation.

We have already discussed rotational alignment sensitivity in detail. For the other types of misalignment, we may summarize the results briefly as follows.

First, consider reference-beam angle misalignment. In an angle-multiplexing volume-holographic memory, misalignment due to reference-beam angle errors along the Bragg-selectivity direction (i.e., the direction in which the reference beam is scanning) may cause reduction of the signal strength, excessive cross talk, or even reconstruction of the incorrect hologram (page). In contrasts, angle errors perpendicular to the Bragg-selectivity direction will cause the reconstructed image to shift in 
Fourier-plane holograms but not in image-plane holograms. In this case image-plane holograms are less sensitive than FT type holograms.

For wavelength-multiplexing systems, the configuration commonly used has the signal beam and the reference beam counterpropagating. In this case the Bragg selectivity is at a minimum (it is zero to first order). This result implies that for FT-type holograms any referencebeam angle error will cause the reconstructed image to shift.

Next, consider the effect of reference-beam wavelength misalignment. In a wavelength-multiplexing volumeholographic memory with the usual counterpropagating geometry, error in the wavelength may cause the incorrect hologram (page) to be read out. The reconstructed image, however, does not shift. For angle-multiplexing FT-type systems, error in the reference-beam wavelength may cause signal loss or cross talk or may cause the wrong page to be read out, the reconstructed image to shift, or both. For angle-multiplexing Fresnel holograms, error in the wavelength results in displacement of the reconstructed image and, in general, in defocusing of the image. ${ }^{15}$ The defocusing effect is zero for image-plane holograms.

For FT-type holograms, translation (or shift) errors are not a problem because the direction of the reconstructed plane waves does not change, and therefore the reconstructed image (intensity pattern) does not change (although the phase of the pattern does change). For Fresnel holograms (and image-plane holograms in particular), translation does have an effect. Because we would like to store data at high density, the pixels at the hologram are small, and the system is very sensitive to translation errors.

The final source of alignment error is hologram tilt (i.e., the normal of the recording media surface changes direction). When the tilt is along the Bragg-selectivity direction of the volume hologram, the effect is similar to the reference-beam angle's changing directions. When the direction is perpendicular to the Bragg-selectivity direction, the reconstructed image shifts for FT-type holograms. For tilt errors, FT-type holograms are more sensitive than image-plane holograms.

In summary, we have shown that the reconstructed image read out from a hologram will always rotate by the same amount as the hologram itself rotates. What changes is the apparent center of rotation and the radius of rotation, neither of which must be the same as that of the actual hologram. The best that we can do, therefore, is to arrange for the center of rotation to be at the center of the reconstructed image. Although rotation is perpendicular to the Bragg-selectivity direction, it does affects image reconstruction, either partially obscuring the image or edge enhancing the image. We have also shown that it is possible to achieve maximum storage density and minimum rotational alignment sensitivity simultaneously. Under the condition of maximum storage density, we have compared the alignment sensitivities of the three recording configurations (image-plane, Fourierplane, and optimum-configuration holograms). The re- sults show that the optimum configuration produces considerable improvement (in terms of alignment sensitivity) compared with image-plane holograms, but the improvement over Fourier-plane holograms is not so dramatic.

\section{ACKNOWLEDGMENTS}

The support of the U.S. Air Force Office of Scientific Research for this work is gratefully acknowledged. We thank A. Yamamura and M. Neifeld, who have contributed greatly to our understanding of alignment problems with parallel readout from two-dimensional optical disks. We also thank the reviewers for their comments and suggestions.

*Present address, MicroDevice Laboratory, Polaroid Corporation, 750 Main Street, M.S. 3-G, Cambridge, Massachusetts 02139 .

\section{REFERENCES}

1. F. H. Mok, D. Psaltis, and G. W. Burr, "Spatial and anglemultiplexing holographic random access memory" in Photonics for Computers, Neural Networks, and Memories, S. T. Kowel, W. J. Miceli, and J. A. Neff, eds., Proc. Soc. PhotoOpt. Instrum. Eng. 1773, 1-12 (1992).

2. F. H. Mok, "Angle-multiplexed storage of 5,000 holograms in lithium niobate," Opt. Lett. 18, 915-917 (1993).

3. L. d'Auria, J. P. Huignard, C. Slezak, and E. Spitz, "Experimental holographic read-write memory using 3-D storage," Appl. Opt. 13, 808-818 (1974).

4. D. Psaltis, "Parallel optical memories," Byte 17, 179-182 (1992).

5. S. Tao, D. R. Selviah, and J. E. Midwinter, "Spatioangular multiplexed storage of 750 holograms in an $\mathrm{Fe}: \mathrm{LiNbO}_{3}$ crystal," Opt. Lett. 18, 912-914 (1993).

6. F. H. Mok, G. W. Burr, and D. Psaltis, "Angle and space multiplexed holographic random access memory (HRAM)," Opt. Mem. Neural Networks 3, 119-127 (1994).

7. H.-Y. S. Li and D. Psaltis, "Three-dimensional holographic disks," Appl. Opt. 33, 3764-3774 (1994).

8. J. J. Amodei and D. L. Staebler, "Holographic recording in lithium niobate," RCA Rev. 33, 71-93 (1972).

9. F. T. S. Yu, S. D. Wu, A. W. Mayers, and S. M. Rajan, "Wavelength multiplexed reflection matched spatial filters using $\mathrm{LiNbO}_{3}$," Opt. Commun. 81, 343-347 (1991).

10. G. A. Rakuljic, V. Leyva, and A. Yariv, "Optical-data storage by using orthogonal wavelength-multiplexed volume holograms," Opt. Lett. 17, 1471-1473 (1992)

11. J. E. Ford, Y. Fainman, and S. H. Lee, "Array interconnection by phase-coded optical correlation," Opt. Lett. 15, 1088-1090 (1990)

12. C. Denz, G. Pauliat, G. Roosen, and T. Tschudi, "Volume hologram multiplexing using a deterministic phase encoding method," Opt. Commun. 85, 171-176 (1991).

13. K. Curtis and D. Psaltis, "Cross talk in phase-coded holographic memories,” J. Opt. Soc. Am. A 10,2547-2550 (1993)

14. D. Brady, K. Hsu, and D. Psaltis, "Periodically refreshed multiply exposed photorefractive holograms," Opt. Lett. 15, 817-819 (1990).

15. H. Kogelnik, "Coupled wave theory for thick hologram gratings,” Bell Syst. Tech. J. 48, 2909-2947 (1969).

16. H.-Y. S. Li, "Photorefractive 3-D disks for optical data storage and artificial neural networks," Ph.D. dissertation (California Institute of Technology, Pasadena, Calif., 1994).

17. A. VanderLugt, "Packing density in holographic systems," Appl. Opt. 14, 1081-1087 (1975). 